Time-resolved photoluminescence spectroscopy of ligand-capped PbS nanocrystals

This article has been downloaded from IOPscience. Please scroll down to see the full text article.

2005 Nanotechnology 16175

(http://iopscience.iop.org/0957-4484/16/2/001)

The Table of Contents and more related content is available

Download details:

IP Address: 150.203.243.41

The article was downloaded on 04/03/2010 at 04:14

Please note that terms and conditions apply. 


\title{
Time-resolved photoluminescence spectroscopy of ligand-capped PbS nanocrystals
}

\author{
Jamie H Warner, Elizabeth Thomsen, Andrew R Watt, \\ Norman R Heckenberg and Halina Rubinsztein-Dunlop
}

Center for Quantum Computer Technology, School of Physical Sciences, University of Queensland, QLD 4072, Australia

E-mail: warner@physics.uq.edu.au

Received 9 November 2004, in final form 30 November 2004

Published 23 December 2004

Online at stacks.iop.org/Nano/16/175

\begin{abstract}
$\mathrm{PbS}$ nanocrystals are synthesized using colloidal techniques and have their surfaces capped with oleic acid. The absorption band edge of the $\mathrm{PbS}$ nanocrystals is tuned between 900 and $580 \mathrm{~nm}$. The PbS nanocrystals exhibit tuneable photoluminescence with large non-resonant Stokes shifts of up to $500 \mathrm{meV}$. The magnitude of the Stokes shift is found to be dependent upon the size of $\mathrm{PbS}$ nanocrystals. Time-resolved photoluminescence spectroscopy of the PbS nanocrystals reveals that the photoluminescence has an extraordinarily long lifetime of $1 \mu \mathrm{s}$. This long fluorescence lifetime is attributed to the effect of dielectric screening similar to that observed in other IV-VI semiconductor nanocrystals.
\end{abstract}

\section{Introduction}

Semiconductor nanocrystals (NCs) have become immensely popular due to the ability to tailor their optical and energetic properties by varying the size [1-4], shape [5, 6] and material [7-11]. Quantum confinement of charge carriers in nanocrystals whose size is comparable to their excitonic Bohr radius results in discrete energy levels and narrow optical transitions. Nanocrystals are currently exploited in a range of applications ranging from biological imaging, quantum computing, and solar cells to electroluminescent devices [12-17].

An increase in the quantum confinement of charge carriers generally leads to more atomic-like behaviour in nanocrystals and this is appealing for almost all applications. $\mathrm{PbS}$ nanocrystals have charge carriers with equal effective masses and an exciton Bohr radius of $18 \mathrm{~nm}$ [18]. Strong quantum confinement of both charge carriers can be easily achieved by synthesizing $\mathrm{PbS}$ nanocrystals with radius less than $18 \mathrm{~nm}$ using many of the existing colloidal techniques $[19,20]$. Thus $\mathrm{PbS}$ nanocrystals offer the unique opportunity of studying semiconductor nanocrystals in the strong confinement regime.

A recent rapid rise in the research interests of colloidal $\mathrm{PbS}$ nanocrystals has been spawned by the ability to generate high photoluminescence yields [21-26]. Hines et al used organic ligands to control $\mathrm{PbS}$ nanocrystal growth in organic solvents and also passivate the surface [21]. The passivation of the $\mathrm{PbS}$ nanocrystal surface states enabled photoluminescence from the $\mathrm{PbS}$ nanocrystal band edge with quantum yields of up to $20 \%$ [21].

The high photoluminescence yields and the ability to tune the size have led to $\mathrm{PbS}$ nanocrystals being incorporated into novel electroluminescence devices consisting of blended nanocrystals and conducting polymers $[23,24]$. These devices hold great promise for being efficient emitters in the nearIR region, especially the telecommunications region [23, 24]. Improvements in the performance of such opto-electronic devices require a detailed understanding of the energy relaxation mechanisms in the $\mathrm{PbS}$ nanocrystals.

Here we present a method for tuning the band edge of oleic-acid-capped PbS nanocrystals from 900 to $580 \mathrm{~nm}$, with a size change from 2 to $1 \mathrm{~nm}$ respectively. The $\mathrm{PbS}$ nanocrystals with an absorption band edge at $580 \mathrm{~nm}$ exhibit photoluminescence with a peak at $770 \mathrm{~nm}$ and this has allowed time-resolved photoluminescence spectra for oleic-acid-capped $\mathrm{PbS}$ nanocrystals to be obtained. Time-resolved photoluminescence spectroscopy provides information about the nature of energy relaxation in the system, 


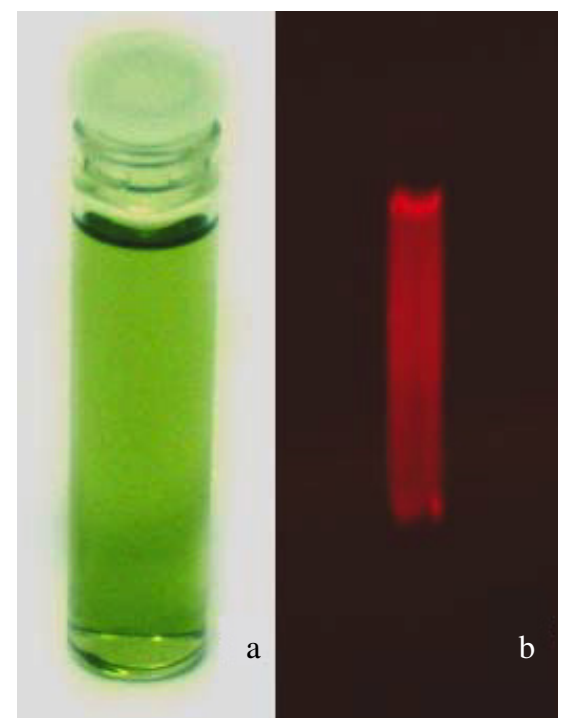

Figure 1. (a) Image of a vial of oleic-acid-capped PbS NCs prepared at $40^{\circ} \mathrm{C}$, (b) fluorescence image of a vial of oleic-acid-capped PbS NCs excited by a UV lamp.

helping distinguish between direct exciton recombination and relaxation with the assistance of trap states or phonons. Steady state absorption and photoluminescence spectroscopy is also used to examine the changes in the non-resonant Stokes shift as a function of nanocrystal size.

\section{Experiment}

$\mathrm{PbS}$ nanocrystals were prepared in a similar manner to Hines et al, but with $\mathrm{H}_{2} \mathrm{~S}$ gas used instead of bis(trimethylsilyl)sulfide (TMS). This enabled synthesis temperatures as low as $40^{\circ} \mathrm{C}$ and consequently smaller $\mathrm{PbS}$ nanocrystals were formed.

A typical reaction is as follows: in a continuously stirred sealed flask, a lead oleate precursor solution containing $0.1 \mathrm{~g}$ lead acetate, $10 \mathrm{ml}$ of $\mathrm{n}$-decane and $0.4 \mathrm{ml}$ oleic acid was heated to $130^{\circ} \mathrm{C}$ for $20 \mathrm{~min}$ under a continuous flow of argon. Next the solution was cooled to $40^{\circ} \mathrm{C}$ and $1 \mathrm{ml}$ of $\mathrm{H}_{2} \mathrm{~S}$ gas was injected at a rate of $1 \times 10^{-4} 1 \mathrm{~s}^{-1}$ into the gas phase of the sealed flask. $\mathrm{PbS}$ nanocrystal formation occurred within seconds of the complete injection of the $\mathrm{H}_{2} \mathrm{~S}$ gas and after 1 min the reaction was complete and the solution was subsequently cooled back to room temperature. This procedure produced a colloidal suspension of oleic-acid-capped PbS NCs with a mean size of approximately 1-2 $\mathrm{nm}$ (as determined by HRTEM) and a band edge at $580 \mathrm{~nm}$. Such NC solutions can be readily diluted in a non-polar solvent such as toluene or hexane.

Fine control of the mean $\mathrm{NC}$ size was achieved by adjusting the temperature between 40 and $130^{\circ} \mathrm{C}$, before the $\mathrm{H}_{2} \mathrm{~S}$ gas was added to induce $\mathrm{NC}$ formation. This allowed the absorption band edge to be tuned between 580 and $900 \mathrm{~nm}$.

The CdSe semiconductor nanocrystals used in the time-resolved photoluminescence spectra were commercial (Qdot company) $5 \mathrm{~nm} \mathrm{CdSe/ZnS} \mathrm{core-shell} \mathrm{NCs} \mathrm{with} \mathrm{a}$ room temperature quantum yield of up to $50 \%$ and a photoluminescence lifetime of $10 \mathrm{~ns}$.

Room temperature optical absorption spectra were obtained with a Perkin Elmer Lambda 40 UV/vis spectrometer
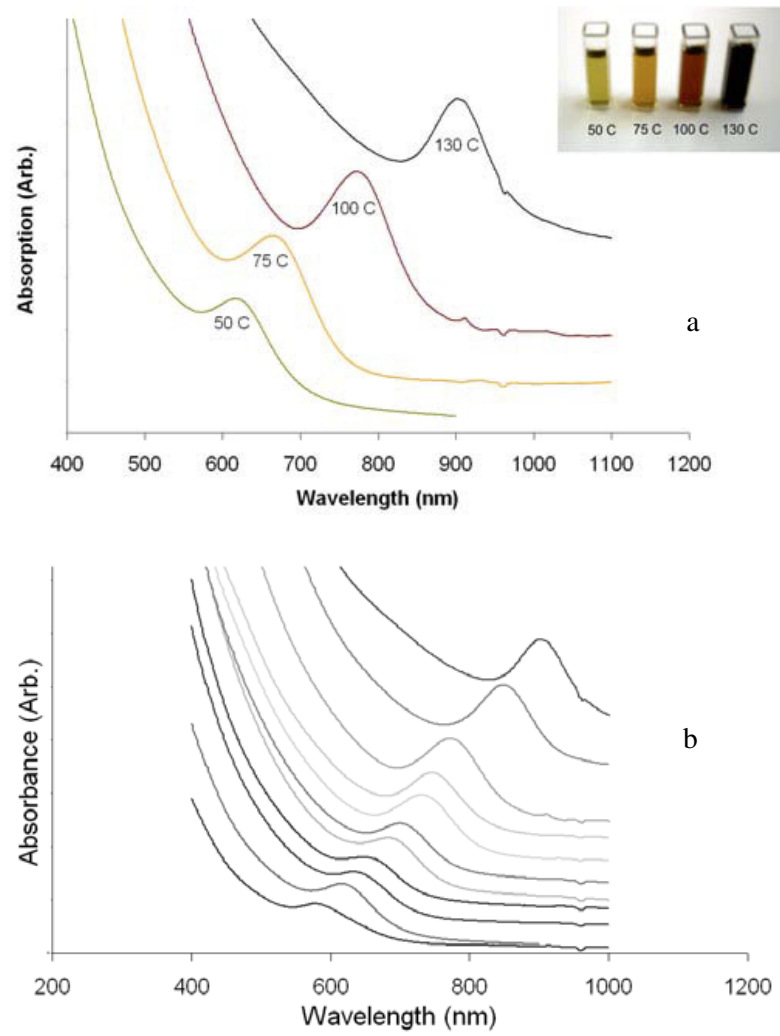

Figure 2. (a) Absorption spectra of oleic-acid-capped $\mathrm{PbS}$ nanocrystals prepared at $50,75,100$ and $130{ }^{\circ} \mathrm{C}$. (b) Fine tuning of the absorption spectra of oleic-acid-capped $\mathrm{PbS}$ nanocrystals as the temperature is varied between 40 and $130{ }^{\circ} \mathrm{C}$.

operating with the slits set to a $2 \mathrm{~nm}$ band pass and $1 \mathrm{~nm}$ grating steps. Room temperature photoluminescence spectra were collected perpendicular to an excitation laser beam (532 nm CW) using a single lens, coupled into a single-grating (1200 $\mathrm{g} \mathrm{mm}^{-1}$ ) monochromator (Acton Research) with a silicon photodiode detector. Time-resolved photoluminescence decays were obtained using a timecorrelated single-photon counting spectrometer (Picoquant Fluotime 200) with $400 \mathrm{~nm}$ wavelength $100 \mathrm{fs}$ pulses sourced from a frequency-doubled mode-locked Ti:sapphire laser (Spectra Physics Tsunami). Pulse repetition rates of $1 \mathrm{~Hz}$ were used and the limit of the apparatus's observation window was $1 \mu \mathrm{s}$.

\section{Results and discussion}

Figure 1(a) shows an image of a vial of the smallest oleicacid-capped $\mathrm{PbS}$ nanocrystals and figure 1(b) shows this vial fluorescing red when excited with a UV lamp. The absorption band edge of this solution was at $580 \mathrm{~nm}$ and the striking green colour is remarkable for a colloidal suspension of $\mathrm{PbS}$ nanocrystals.

In figure 2(a) we see the absorption spectra for four $\mathrm{PbS}$ nanocrystal solutions synthesized with increasing temperature. The standard deviation of the position of the band edge peak for a specific synthesis temperature was typically $5 \%$. This is mainly due to the difficulties in the control of the addition rate of $\mathrm{H}_{2} \mathrm{~S}$. Figure 2(b) shows the ability to tune the band 
Time-resolved photoluminescence spectroscopy of ligand-capped PbS nanocrystals
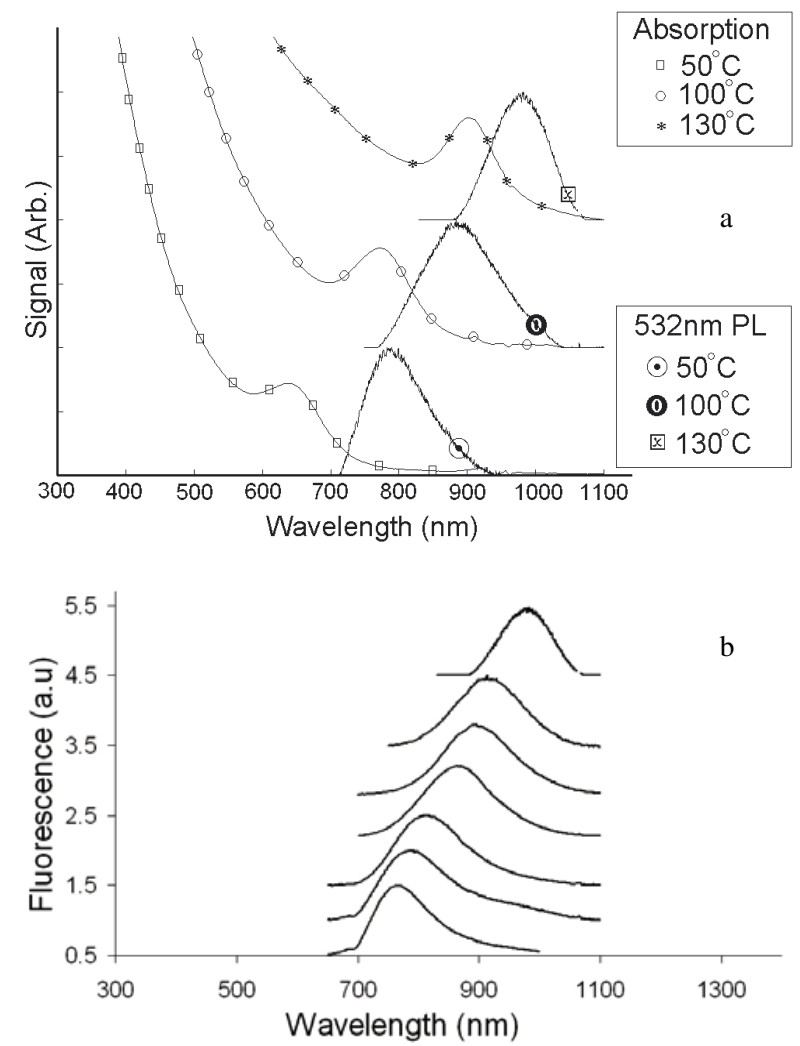

Figure 3. (a) Photoluminescence spectra of oleic-acid-capped PbS nanocrystals prepared at $50,75,100$ and $130^{\circ} \mathrm{C}$. (b) Fine tuning of the photoluminescence spectra of oleic-acid-capped $\mathrm{PbS}$ nanocrystals as the temperature is varied between 40 and $130^{\circ} \mathrm{C}$.

edge of the absorption spectrum anywhere between 580 and $900 \mathrm{~nm}$ as the temperature is varied between 40 and $130^{\circ} \mathrm{C}$. The band edge remains a clearly defined peak that suggests the size distribution remains narrow even as the mean size is reduced. Clearly the band edge shifts to lower energies as the synthesis temperature is increased. This suggests that lower synthesis temperatures yield the smallest $\mathrm{PbS}$ nanocrystals.

Figure 3(a) shows the absorption and photoluminescence spectra of PbS nanocrystal solutions synthesized at 50, 100 and $130^{\circ} \mathrm{C}$. We see that, as the absorption spectrum is tuned, so too is the photoluminescence. The spectral bandwidths of the photoluminescence spectra in figure 3(a) are comparable to the absorption peaks, suggesting that the emission is from a single state rather than an ensemble of trap states [2]. Trapped state emission from an NC solution typically has a full width at half maximum (FWHM) significantly larger than the FHWM of a Gaussian fitted to the band edge peak in the absorption spectrum. The photoluminescence spectra presented in figure 3(b) show the ability to tune the emission from 770 to $1000 \mathrm{~nm}$ as the temperature is increased from 40 to $130^{\circ} \mathrm{C}$. The spectral width of the photoluminescence remains fairly constant as the peak shifts from 770 to $1000 \mathrm{~nm}$.

The clearly defined band edge peaks in the absorption spectra of the oleic-acid-capped $\mathrm{PbS}$ nanocrystals allowed relatively precise measurements of the non-resonant Stokes shift. Figure 4 shows the variation of the non-resonant Stokes shift as a function of the absorption band edge peak. It is well known that in PbS NCs the absorption band edge depends

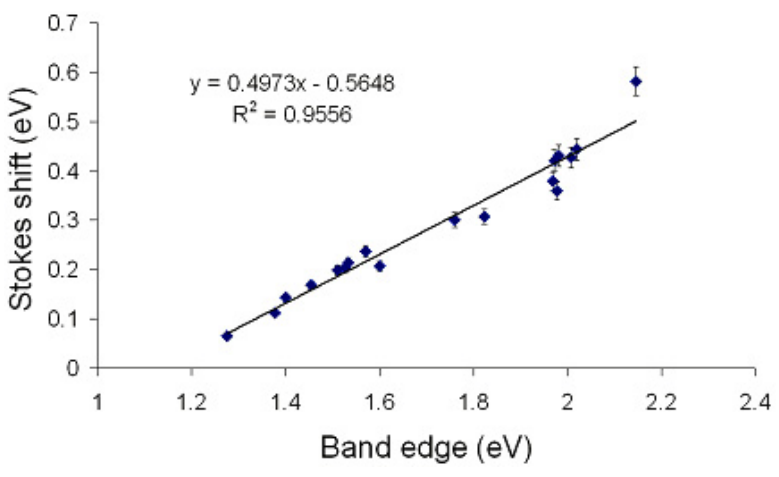

Figure 4. Variation of Stokes shift as a function of the absorption band edge of oleic-acid-capped $\mathrm{PbS}$ nanocrystals.

upon the nanocrystal size, with the band edge shifting to higher energies as the size of the nanocrystal decreases [18,21,27]. At these extremely small nanocrystal sizes, tiny deviations in the mean size of the PbS NCs lead to a large shift in the absorption band edge [27]. TEM analysis also correlated this but could only be used to show size shifts between the smallest and largest nanocrystal samples. Thus by correlating the Stokes shift with the absorption band edge we have a more accurate description of how the Stokes shift might change with the size and energy level structure of the oleic-acid-capped PbS NCs.

In figure 4 we see that the Stokes shift increases linearly as the band edge increases in energy. This means that as the size of the PbS nanocrystal decreases the Stokes shift increases. Non-resonant Stokes shifts have also been reported for other NCs, such as PbSe [2] (90 meV), CdSe [28] (300 meV) and ZnSe [29], which are comparable with the 100-500 meV measured here for the PbS NCs. For CdSe and ZnSe, the Stokes shift is attributed to a dark exciton state whereby phonons assist excitons from the excited state to a normally spin forbidden state of lower energy, from which they relax to the ground state, emitting a photon of lower energy than the initial absorbing state [28, 29]. However, for $\mathrm{PbSe}$ and $\mathrm{PbS}$ no such state has been theoretically predicted and the nature of the Stokes shift requires further investigation.

The smallest $\mathrm{PbS}$ nanocrystals, synthesized at $40^{\circ} \mathrm{C}$, displayed an absorption band edge at $580 \mathrm{~nm}$ and a photoluminescence peak at $770 \mathrm{~nm}$. Figure 5(a) shows a transmission electron microscopy (TEM) image of the oleicacid-capped $\mathrm{PbS}$ NCs synthesized at $40^{\circ} \mathrm{C}$ and figure $5(\mathrm{~b})$ shows a TEM image of $\mathrm{PbS}$ NCs synthesized at $130^{\circ} \mathrm{C}$. The TEM images in figure 5 show 1 and $2 \mathrm{~nm} \mathrm{PbS}$ nanocrystals for 40 and $130{ }^{\circ} \mathrm{C}$ respectively with a relatively narrow size distribution. The size of these $\mathrm{PbS}$ nanocrystals is extremely small and this leads to a high degree of quantum confinement in these structures. The ability to tune the emission of the oleic-acid-capped $\mathrm{PbS}$ nanocrystals into the near visible regime allows conventional time-resolved photoluminescence spectroscopy measurements to be performed and compared directly to other semiconductor nanocrystals such as CdSe.

Figure 6 shows the time-resolved photoluminescence spectra from a colloidal solution of $1 \mathrm{~nm}$ oleic-acid-capped $\mathrm{PbS}$ NCs, synthesized at $40^{\circ} \mathrm{C}$, and as a comparison a colloidal solution of $5 \mathrm{~nm} \mathrm{CdSe} / \mathrm{ZnS}$ nanocrystals. The fluorescence decays in figure 6 were recorded at the respective peaks in the emission of the two samples. The lifetime of the $\mathrm{PbS}$ 

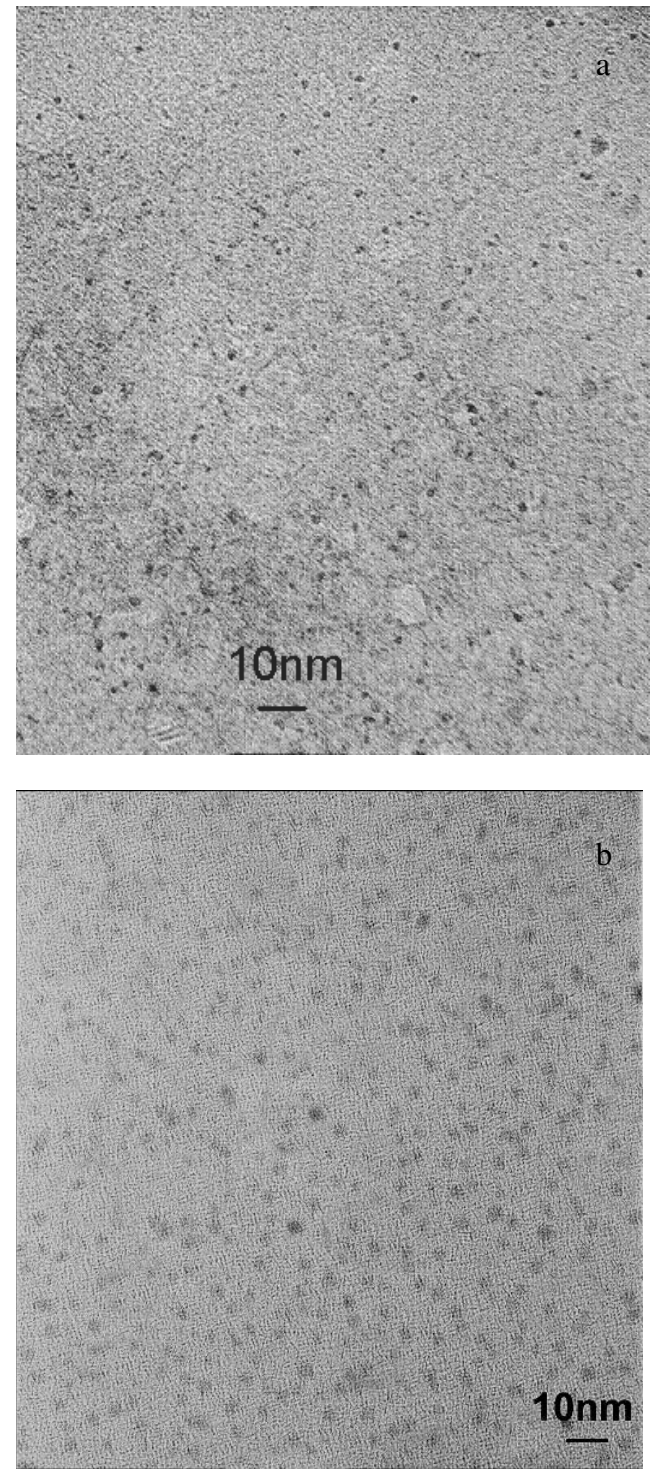

Figure 5. Transmission electron microscopy image of oleic-acid-capped $\mathrm{PbS}$ nanocrystals synthesized at (a) $40^{\circ} \mathrm{C}$ and (b) $130^{\circ} \mathrm{C}$.

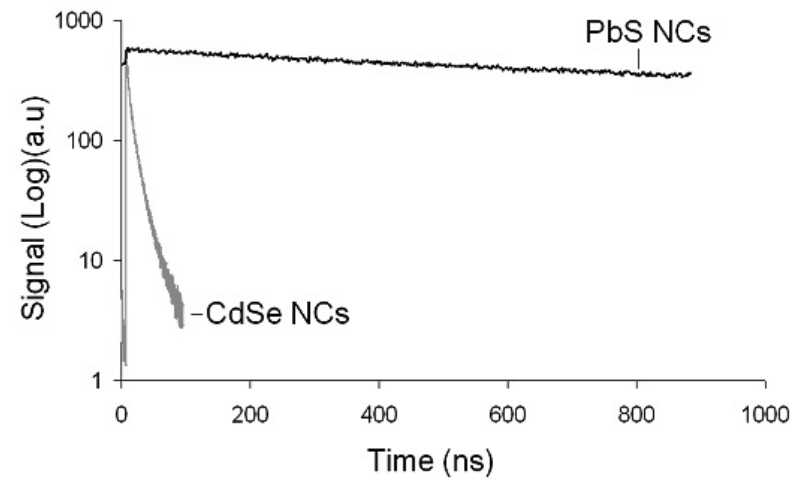

Figure 6. Time-resolved photoluminescence lifetime spectra of $1 \mathrm{~nm}$ oleic-acid-capped PbS nanocrystals and $5 \mathrm{~nm}$ CdSe nanocrystals.

nanocrystals has a single-exponential time constant measured to be $1 \mu \mathrm{s}$ and is two orders of magnitude longer than the
$\mathrm{CdSe} / \mathrm{ZnS}$ nanocrystals. The observed decay time of the $\mathrm{PbS}$ $\mathrm{NCs}$ is longer than expected for a dipole transition, but shorter than expected for emission related to trap states [30].

Colloidal $\mathrm{PbSe}$ semiconductor nanocrystals have also shown long fluorescence lifetimes of up to $0.88 \mu \mathrm{s}$, similar to the $\mathrm{PbS}$ nanocrystals measured here [30]. The long radiative lifetimes in the PbSe nanocrystals were attributed to the effects of dielectric screening and there was no need to invoke a triplet or quasi-forbidden transitions, such as a dark exciton state, to explain the results [30]. The screening of the radiating field inside the nanocrystal has the effect of weakening the internal field and consequently increases the radiative lifetime by a factor of $\left[3 \varepsilon_{1} /\left(\varepsilon_{2}+2 \varepsilon_{1}\right)\right]^{-2}$, where $\varepsilon_{1} \sim 1.88$ and $\varepsilon_{2} \sim 23$ are the optical dielectric constants of the solvent host (hexane) and the nanocrystal material $(\mathrm{PbS})$ respectively $[30,31]$. This leads to an increase of the radiative lifetime by a factor of 22 , which is a $15 \%$ increase in this factor as compared to $\mathrm{PbSe} \mathrm{NCs}$ in chloroform in [30]. This also correlates with the $1 \mu$ s lifetime of the PbS NCs being 12\% longer than the $0.88 \mu$ s lifetime of the PbSe NCs. The long lifetimes associated with the effect of dielectric screening is predicted for all the IV-VI semiconductor nanocrystals with high dielectric constants [30]. Thus the long lifetimes measured in the colloidal $\mathrm{PbS}$ nanocrystals are not unexpected.

\section{Conclusion}

In conclusion, we have shown the ability to tune the $\mathrm{PbS} \mathrm{NC}$ absorption band edge from 900 to $580 \mathrm{~nm}$ with decreasing temperature. The photoluminescence from the $\mathrm{PbS}$ NCs also shifted with the absorption band edge from 1000 to $770 \mathrm{~nm}$. TEM confirmed the size shifts in the PbS NCs and the photoluminescence lifetime of $1 \mathrm{~nm}$ oleic-acid-capped $\mathrm{PbS}$ nanocrystals was measured to be $1 \mu \mathrm{s}$. This long fluorescence lifetime was two orders of magnitude longer than CdSe nanocrystals. The extremely long lifetimes in the $\mathrm{PbS}$ nanocrystals are expected due to the effect of dielectric screening. Steady-state spectroscopy of the oleicacid-capped $\mathrm{PbS}$ NCs revealed large non-resonant Stokes shifts that increased as the nanocrystal size decreased.

Further work to measure the fluorescence lifetimes as a function of nanocrystal size is underway. This should provide more information about the nature of the relatively large nonresonant Stokes shifts observed in these $\mathrm{PbS}$ nanocrystals.

\section{Acknowledgments}

We thank the Australian Research Council for their financial support and Drs M Fernee and S Cooper for their valuable discussions.

\section{References}

[1] Kim S, Park J, Jang Y, Chung Y, Hwang S and Hyeon T 2003 Nano Lett. 31289

[2] Du H, Chen C, Krishnan R, Krauss T D, Harbold J M, Wise F W, Thomas M G and Silcox J 2002 Nano Lett. 2 1321

[3] Alivisatos A P 1996 J. Phys. Chem. 10013226

[4] Rogach A, Kershaw S, Burt M, Harrison M, Kornowski A, Eychmuller A and Weller H 1999 Adv. Mater. 11552 
Time-resolved photoluminescence spectroscopy of ligand-capped PbS nanocrystals

[5] Lee S-M, Jun Y, Cho S-N and Cheon J 2002 J. Am. Chem. Soc. $\mathbf{1 2 4} 11244$

[6] Manna L, Scher E C and Alivisatos A P $2000 \mathrm{~J}$. Am. Chem. Soc. 12212700

[7] Nenadovic M T, Comor M I, Vasic V and Micic O I 1990 J. Phys. Chem. 946390

[8] Gallardo S, Gutierrez M, Henglein A and Janata E 1989 Ber Bunsenges. Phys. Chem. 931080

[9] Wuister S F and Meijerink A 2003 J. Lumin. 102/103 338

[10] Wageh S, Shu-Man L, You F T and Xu-Rong X 2003 J. Lumin. 102/103 768

[11] Murray C B, Norris D J and Bawendi M G 1993 J. Am. Chem. Soc. 1158706

[12] Sun B, Marx E and Greenham N C 2003 Nano Lett. 3961

[13] Watt A, Thomsen E, Meredith P and Rubinsztein-Dunlop H 2004 Chem. Commun. 2334

[14] Larson D R, Zipfel W R, Williams R M, Clark S W, Bruchez M P, Wise F W and Webb W W 2003 Science 300 1434

[15] Imamoglu A, Awschalom D D, Burkard G, DiVincenzo D P Loss D, Sherwin M and Small A 1999 Phys. Rev. Lett. 83 4204

[16] Redi F X, Cho K S, Murray C B and O'Brien S 2003 Nature 423968

[17] Haremza J M, Hahn M A, Krauss T D, Chen S and Calcines J 2002 Nano Lett. 21253
[18] Wise F W 2000 Acc. Chem. Res. 33773

[19] Gurin V S 1998 J. Cryst. Growth 191161

[20] Zhou Y, Itoh H, Uemura T, Naka K and Chujo Y 2002 Langmuir $\mathbf{1 8} 5287$

[21] Hines M A and Scholes G D 2003 Adv. Mater. 151844

[22] Chang T-W F, Musikhin S, Bakueva L, Levina L, Hines M A, Cyr P W and Sargent E H 2004 Appl. Phys. Lett. 844295

[23] Bakueva L, Musikhin S, Hines M A, Chang T-W F, Tzolov M, Scholes G D and Sargent E H 2003 Appl. Phys. Lett. 82 2895

[24] Bakueva L, Konstantatos G, Levina L, Musikhin S and Sargent E H 2004 Appl. Phys. Lett. 843459

[25] Bakueva L, Konstantatos G, Musikhin S, Ruda H E and Shik A 2004 Appl. Phys. Lett. 853567

[26] McDonald S A, Cyr P W, Levina L and Sargent E H 2004 Appl. Phys. Lett. 852089

[27] Kang I and Wise F W 1997 J. Opt. Soc. Am. B 141632

[28] Liu S-M, Guo H-Q, Zhang Z-H, Li R, Chen W and Wang Z-G 2000 Physica E 8174

[29] Nikesh V V and Mahamuni S 2001 Semicond. Sci. Technol. 16 687

[30] Wehrenberg B L, Wang C and Guyot-Sionnest P 2002 J. Phys. Chem. B 10610634

[31] Bergman D J and Stockman M I 2003 Phys. Rev. Lett. 90 027402 